International Journal of Distributed and Parallel Systems (IJDPS) Vol.3, No.3, May 2012

\title{
PEAK-TO-AVERAGE POWER REDUCTION IN MIMO- OFDM SYSTEMS USING SUB-OPTIMAL ALGORITHM
}

\author{
K Srinivasarao ${ }^{1}$, Dr B Prabhakararao ${ }^{2}$, and Dr M V S Sairam ${ }^{3}$ \\ ${ }^{1}$ Department of Electronics and Communication Engineering, G.V.P College of \\ Engineering for Women, Visakhapatnam, ksrinivas.ece@gmail.com \\ ${ }^{2}$ J.N.T University, Kakinada, drbpr@rediffmail.com \\ ${ }^{3}$ G.V.P. College of Engineering, sairammvs3egmail.com
}

\begin{abstract}
Multiple-input multiple-output orthogonal frequency division multiplexing (MIMO-OFDM) technology is one of the most attractive candidates for fourth generation (4G) mobile radio communication. It effectively combats the multipath fading channel and improves the bandwidth efficiency. At the same time, it also increases system capacity so as to provide a reliable transmission. However, the main drawback of MIMO-OFDM system is high peak-to-average power ratio (PAPR) for large number of sub-carriers, which result in many restrictions for practical applications. Coding, phase rotation and clipping are among many PAPR reduction schemes that have been proposed to overcome this problem. In this paper, we mainly investigate the PAPR reduction performance with two different PAPR reduction methods: partial transmit sequence (PTS) and selective mapping (SLM). These two methods are sub-entities of phase rotation scheme. We propose an algorithm, Sub-Optimal algorithm that shows better PAPR reduction compared to the existing algorithms. Results are verified using MATLAB software.
\end{abstract}

\section{KEYWORDS}

Multiple input multiple output (MIMO), orthogonal frequency division multiplexing (OFDM), peak-toaverage power ratio (PAPR), selected mapping (SLM), partial transmit sequence (PTS), Sub-Optimal Algorithm, Complementary cumulative distribution function (CCDF).

\section{INTRODUCTION}

Third generation $(3 \mathrm{G})$ mobile communication Technologies implemented almost around the world, are not able to meet the requirements of the day viz., high data rates nor throughputs. Besides, voice communication in $3 \mathrm{G}$ relies on circuit switching technology, the same as in second-generation (2G) communication systems, rather than pure Internet Protocol (IP) approach. Researchers have been trying for the next evolutionary fourth generation (4G) communication systems to provide a comprehensive and secure IP solution where voice, data, and multimedia can be offered to users at "anytime, anywhere" with higher data rates than previous generations [1]. Since bandwidth resource for $4 \mathrm{G}$ mobile communications is still scarce. To improve spectrum efficiency and achieve higher rates at more than 100Mbps more advanced techniques need to be implemented. The limitation of modulation schemes in existing communication systems has become an obstruction in further increasing the data rate. Hence, next generation mobile communication systems need more sophisticated modulation scheme and information transmission structure. Multiple input multiple outputs (MIMO) and orthogonal frequency division multiplexing (OFDM) have therefore been adopted due to their superior performance. They promise to become key high-speed wireless communication technologies and combining them can provide wireless industry evolution from $3 \mathrm{G}$ to $4 \mathrm{G}$ system. In MIMOOFDM system, the output is the superposition of multiple sub-carriers. In this case, instantaneous power outputs increases and may demand higher powers than the mean power of

DOI : $10.5121 /$ ijdps.2012.3322 
the system since the phases of these carriers are same. High PAPR is one of the most serious problems in MIMO-OFDM system. To transmit signals with high PAPR, it requires power amplifiers with very high power scope. These kinds of amplifiers are very expensive and have low efficiency-cost factor. This gives rise to non-linear distortion which changes the superposition of the signal spectrum resulting in performance degradation. If there are no measures to reduce the high PAPR, MIMO-OFDM system could face serious restriction for practical applications. To combat increase in PAPR, one intuitive solution is to adopt amplifiers to have larger trade-off range. The outline of the paper is as follows. After system description, which is presented in Sect. 2, Description of PAPR, three distinctive types of PAPR reduction techniques are discussed and we focus our research on signal scrambling techniques in Sect. 3. Two sub-type algorithms, selected mapping (SLM) and partial transmit sequence (PTS) are investigated. A comprehensive analysis and comparison are conducted in terms of all possible influencing factors and PAPR reduction performance, respectively and some research findings are reported based on the simulation results in Sect. 4 and conclusions of paper are presented in Sect. 5 .

\section{SYSTEM DESCRIPTION}

\subsection{Basic Structure of OFDM System}

Basic OFDM transmission system is shown in Fig. 1. An OFDM symbol is made of sub-carriers modulated by constellations mapping. This mapping can be achieved from phase-shift keying (PSK) or quadrature amplitude modulation (QAM). For an OFDM system with $\mathrm{N}$ sub-carriers, the high-speed binary serial input stream is denoted as $\left\{a_{i}\right\}$. After serial to parallel (S/P) conversion and constellation mapping, a new parallel signal sequence $\left\{d_{0,1}, \cdots, d_{i}, \cdots, d_{N-1}\right\}$ is obtained, $d_{i}$ is a discrete complex-valued signal. Here, $d_{i} \in \pm 1$ when BPSK mapping is adopted. When QPSK mapping is used, $d_{i} \in\{ \pm 1, \pm j\}$.Each element of parallel signal sequence is supplied to $N$ orthogonal sub-carriers $e^{j^{2} \pi f_{0} t}, e^{j^{2} \pi f_{1} t}, \cdots, e^{j^{2} \pi f_{N-1} t}$ for modulation, respectively. Finally, modulated signals are added together to form an OFDM symbol.

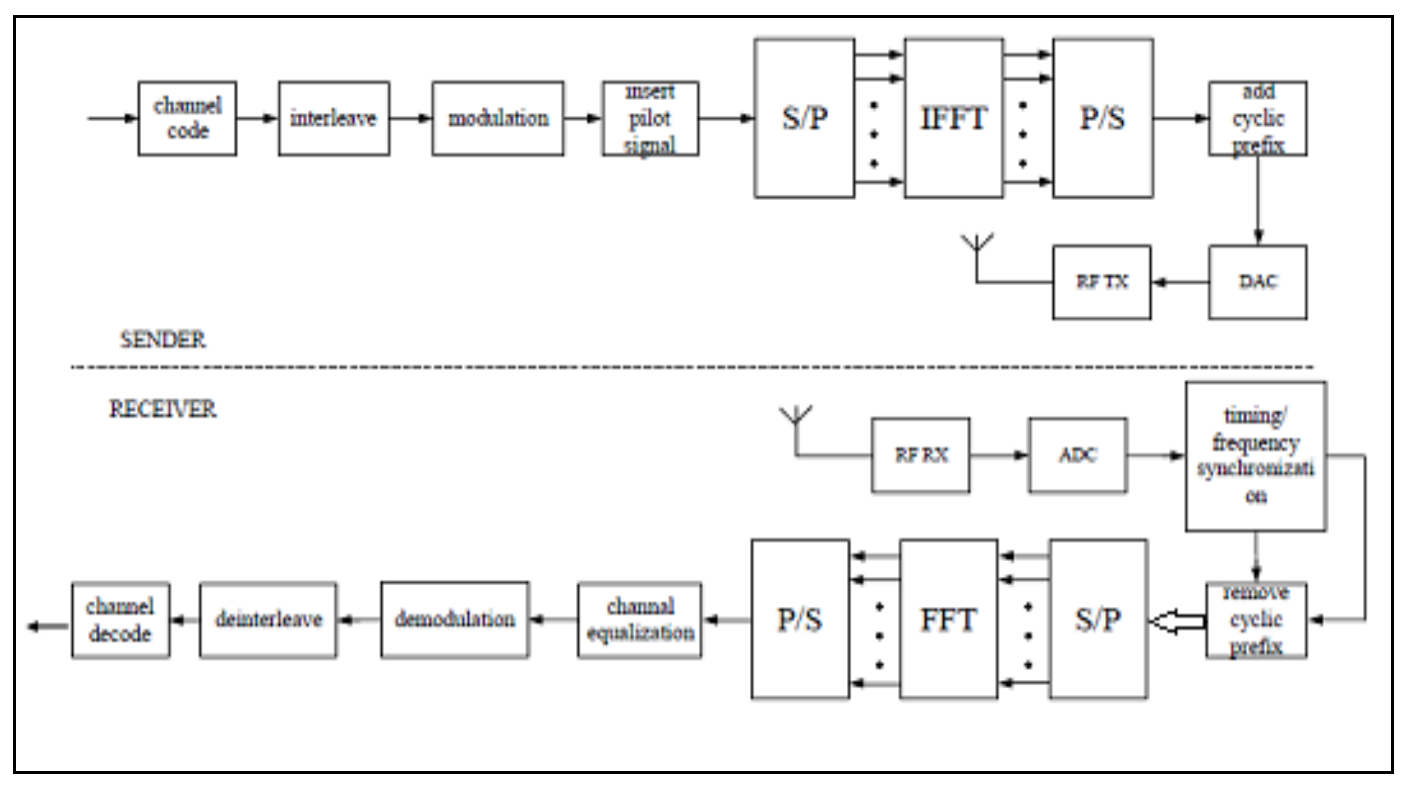

Figure 1 Basic structure of OFDM system. 
The frequency of an OFDM signal can be expressed as

$$
f i=f c+i \cdot \Delta f
$$

where $f c$ stands for carrier frequency, $\Delta f$ is the smallest interval between different sub-carrier frequencies. $\Delta f$ is given by

$$
\Delta f=1 / T=1 / N t s
$$

where $t s$ is time interval of symbol sequence $\left\{d_{0}, d_{1}, \cdots, d_{i}, \cdots, d_{N-1}\right\}$. Generally, we use complex baseband equivalent signal to describe OFDM output signal, which can be expressed as follows:

$$
s(t)=\sum_{i=0}^{N 1} d_{i} e^{j 2 \pi i \Delta f t}=\sum_{i=0}^{N-1} d_{i} e^{j 2 \pi \frac{i}{T} t}, \quad t \in[0, T]
$$

The real and imaginary parts of complex factor corresponding to in-phase components and quadrature components of OFDM symbols, respectively.

\subsection{MIMO-OFDM System}

In high-speed wireless communication, OFDM can be applied to transform frequency-selective MIMO channel into parallel flat MIMO channels, in multipath fading environment also high data rate robust transmission can be achieved by reducing the complexity of the receiver.

\subsubsection{Basic Structure of MIMO-OFDM System}

At the transmitting end, a number of transmission antennas are used. An input data bit stream is supplied into space-time coding, then modulated by OFDM and finally fed to antennas for sending out (radiation). At the receiving end, in-coming signals are fed into a signal detector and processed before recovery of the original signal is made. Fig. 2. shows the basic structure of a MIMO-OFDM system.

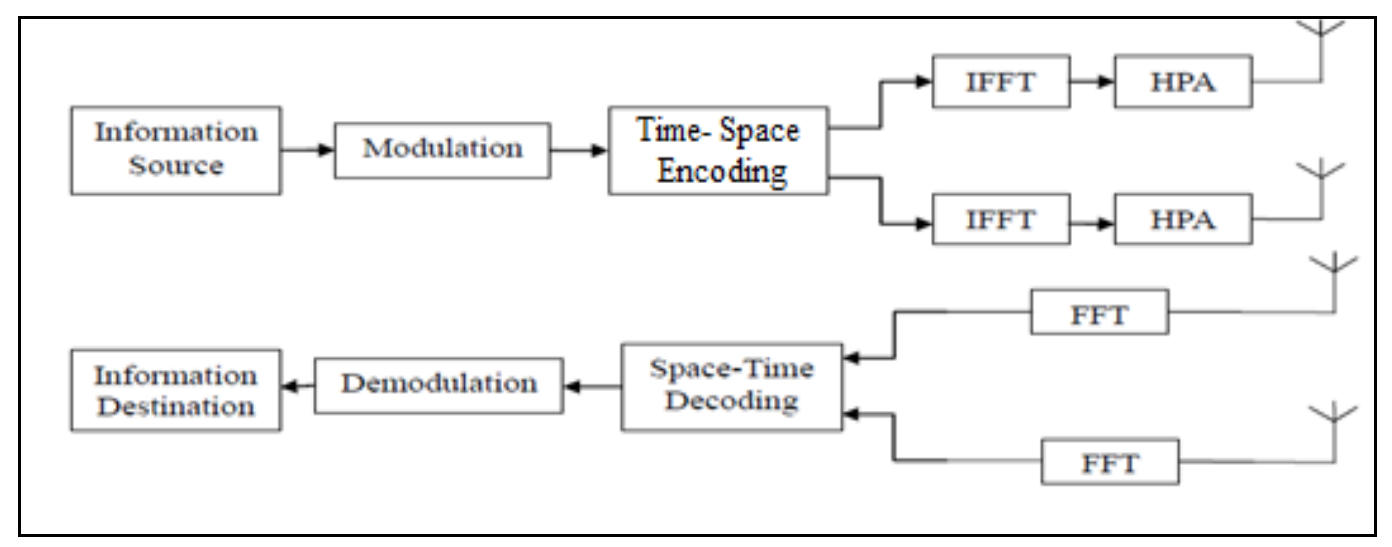

Figure 2 Basic structure of MIMO-OFDM system.

In MIMO-OFDM system, for $k=0, \ldots, K-1, h_{l}^{(q, p)}(n)$ is the impulse response of $p^{\text {th }}$ transmitter antenna to $q^{\text {th }}$ receiver antenna for $l^{\text {th }}$ channel, where $n$ is sequence number of the symbol, and $K$ is the total number of sub-carriers.

Then the frequency response ${ }^{H_{k}^{(q, p)}(n)}$ of $k^{\text {th }}$ sub-carrier can be expressed as follows:

$$
H_{k}^{(q, p)}(n)=\sum_{l=1}^{L-1} h_{l}^{(q, p)}(n) W_{K}^{k l}
$$

Assuming that $W_{K}=e^{-j 2 \pi / K}$ and $M$ and $N$ are the total number of transmitter and receiver antennas, the output response for the $q^{\text {th }}$ receiver antenna can be written as:

$$
y_{k}^{(q)}(n)=\sum_{p=1}^{M} H_{k}^{(q, p)}(n) x_{k}^{(p)}(n)+\zeta_{k}(n)
$$

$q=1 \ldots N$ and $\zeta(n)$ is Gaussian noise with variance $\delta_{n}^{2}$. 


\section{PEAK-TO-AVERAGE POWER RATIO IN OFDM SYSTEM}

The instantaneous output of an OFDM system often has large fluctuations compared to traditional single-carrier systems. This requires that system devices, such as power amplifiers, A/D converters and D/A converters, must have large linear dynamic ranges. If this is not satisfied, the peak signal goes into the non-linear region of devices at the transmitter, such as high out of band radiation and inter-modulation distortion. PAPR reduction techniques are therefore of great importance for OFDM systems [2].

\subsection{PAPR}

Theoretically, large peaks in OFDM system can be expressed as Peak-to-Average Power Ratio, or referred to as PAPR, in some literatures, also written as PAR. It is defined as [3].

$$
P A P R=\frac{P_{\text {peak }}}{P_{\text {average }}}=10 \log _{10} \frac{\max \left[\left|x_{n}\right|^{2}\right]}{E\left[\left|x_{n}\right|^{2}\right]}
$$

Where $P_{\text {peak }}$ represents peak output power, $P_{\text {average }}$ means average output power. [ $\left.\cdot\right]$ denotes the expected value, $x_{n}$ represents the transmitted OFDM signals. Mathematically $x_{n}$ is expressed as:

$$
x_{n}=\frac{1}{\sqrt{N}} \sum_{k=0}^{N-1} X_{k} W_{N}^{n k}
$$

Where $X_{k}$ represents $\mathrm{k}^{\text {th }}$ input symbol.

The PAPR of baseband signal will reach its theoretical maximum at $P A P(d B)=10 \log N$. For example, for a 16 sub-carriers system, the theoretical PAPR value is $12 \mathrm{~dB}$. Fig. 3 . shows the amplitude characteristic of an OFDM system with 16 sub-carriers. According to the graph, it can be seen that the maximum magnitude of the OFDM signals is less than the upper limit value 16 and corresponding PAPR is also lower than the theoretical maximum $12 \mathrm{~dB}$. The special case happens when signal sub-carriers are modulated by symbols which have the same initial phase. Assuming that input binary sequence contains 16 "1s" for the whole sequence. After PSK constellation mapping and IFFT operation, instant power reaches its theoretical maximum. Fig. 3 (b) shows the result when input binary sequence contains 16 " $1 \mathrm{~s}$ ", denoted by [1111111111111111]. In this scenario, the maximum amplitude reaches the value of 16 . The PAPR can be calculated from $P A P(d B)=10 \log N$ and in this case it is $12 \mathrm{~dB}$.

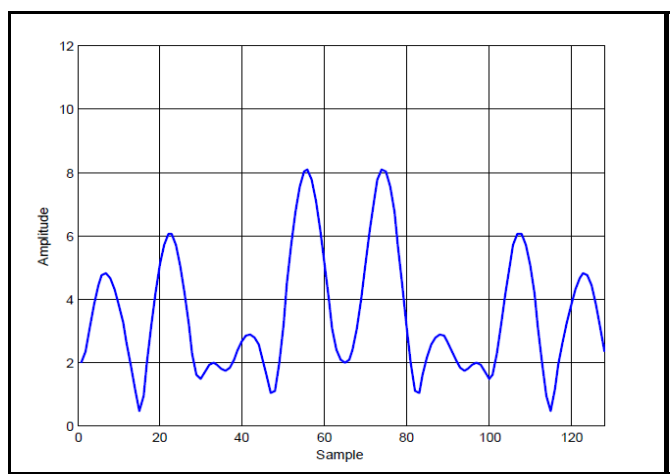

(a)

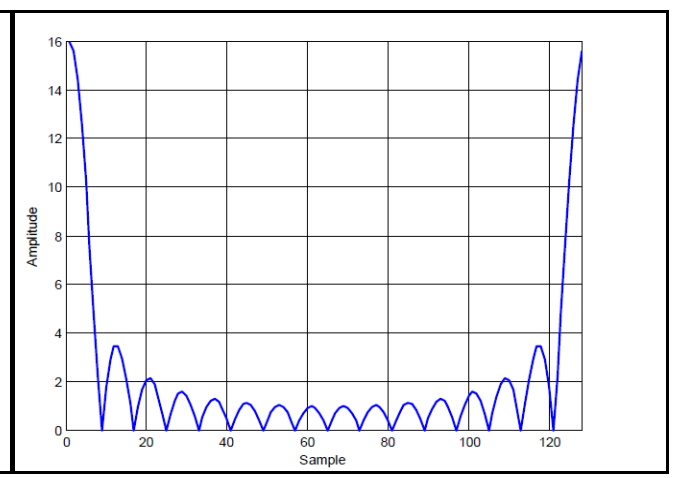

(b)

Figure 3 (a) An OFDM signal waveform in time domain, and (b) High PAPR when sub-carriers are modulated by same symbols.

By observing the simulation result in Fig. 3, we can make a conclusion that the amplitude of OFDM signal reaches its peak value when the input data sequence has a larger consistency. At the same time, the maximum PAPR value will be reached as well. Another commonly used 
parameter is the Crest Factor $(\mathrm{CF})$, which is defined as the ratio between maximum amplitude of OFDM signal $(t)$ and root-mean-square (RMS) of the waveform. The CF is defined as [4]:

$$
C F(s(t))=\frac{\max [|s(t)|]}{E\left[\|\left. s(t)\right|^{2} \mid\right]}=\sqrt{P A P R}
$$

\subsubsection{Probability Distribution Function of PAPR}

According to central limit theorem, for a large number of sub-carriers in multi-carrier signal, the real and imaginary part of sample values in time-domain will obey Gaussian distribution with mean value of 0 and variance of 0.5 . Therefore, the amplitude of multi-carrier signals follows Rayleigh distribution with zero mean and a variance of $N$ times the variance of one complex sinusoid [15]. Its power value obeys a $\chi 2$ distribution with zero mean and 2 degrees of freedom. Cumulative Distribution Function (CDF) is expressed as follows

$$
F(z)=1-\exp (-z)
$$

Assuming that the sampling values of different sub-channels are mutually independent, and free of oversampling operation, the probability distribution function for PAPR less than a certain threshold value, is therefore expressed as

$$
P(P A P R<z)=F(z)^{N}=(1-\exp (-z))^{N}
$$

In practice, it is preferred to take the probability of PAPR exceeding a threshold as measurement index to represent the distribution of PAPR. This can be described as "Complementary Cumulative Distribution Function" (CCDF), and its mathematical expression as

$$
P(P A P R>z)=1-P(P A P R \leq z)=1-F(z)^{N}=1-(1-\exp (-z))^{\mathrm{N}}
$$

\subsection{Reported PAPR Reduction Techniques}

PAPR Reduction solutions can be roughly divided into three categories

1. Signal Distortion: One of the most pragmatic and easiest approaches is clipping and filtering which can snip the signal at the transmitter so as to eliminate the appearance of high peaks above a certain level. Clipping can be implemented to the discrete samples prior to digital-toanalog-convertor (DAC) or by designing analog-to-digital-convertor (ADC) and/or amplifier with saturation levels which are lower than the dynamic range [5]. But due to the nonlinear distortion introduced by this process, orthogonality will be destroyed to some extent which results in serious in band noise and out of band noise.

2. Signal Scrambling Techniques: The fundamental principle of this technique is to scramble each OFDM signal with different scrambling sequences and select one which has the least PAPR value for transmission. Apparently, this technique does not guarantee reduction of PAPR value below a certain threshold, but it can reduce the appearance probability of high PAPR to a great extent. This type of approach includes: Selective Mapping (SLM) and Partial Transmit Sequences (PTS). SLM method applies scrambling rotation to all sub-carriers independently while PTS method only takes scrambling to part of the sub-carriers.

3. Coding Techniques: The core of encoding method is to apply special forward error correction technique to remove the OFDM signals with high PAPR. The classical schemes include linear block code [6], Golay codes and Reed-Muller code [7]. As far as linear block code method is concerned, it is suitable only to the scenario which has a smaller number of sub-carriers. ReedMuller code, high efficiency coding scheme, obtains a lower PAPR for the second order cosets by classifying the Walsh-Hadamard transform (WHT) spectrum of the code words. PAPR can be reduced to $3 \mathrm{~dB}$ with a good error correcting performance with this code. However the encoding method is limited to specific types of constellation. 


\section{RESEARCH ON SIGNAL SCRAMBLING TECHNIQUES}

The emergence of high peak power signal in OFDM system is due to the superposition (IFFT operation) of multiple sub-carrier signals. If multiple sequences which carry the same information are used to represent one transmission process, then the best one can be chosen among those candidates for a given PAPR threshold condition. In this way the occurrence probability of peak power signal can significantly be reduced. The phase rotation method used for reducing the PAPR of OFDM signal is a special case of multiple signal representation (MSR) technology. Its fundamental principle is: Generating multiple signal waveforms which carry the same information and then choose the waveform from those candidates with the smallest PAPR for transmission. This approach reduces the occurrence probability of high peak power signal effectively and optimizes the statistical characteristics of PAPR. This method is one of the non-distortion methods used for reducing PAPR. However, until now, there exist two most effective and meritorious proposals which are called SLM and PTS. In this paper, investigation into these two potent probabilistic approaches is conducted. The two have received so much attention since they provide a low reduction in throughput.

\subsection{Selected Mapping Method}

Rotation PAPR reduction scheme and SLM are shown in Fig. 4. The CCDF of the original signal sequence's PAPR above a threshold $\mathrm{PAPR}_{0}$ is written as $\operatorname{Pr} P A P R>P A P R_{0}$. Thus for $K$ statistical independent signal waveforms, CCDF can be rewritten as $\left[P\left\{P A P R>P A P R_{0}\right\}\right]^{K}$, so that the probability of PAPR that exceeds the same threshold will drop to a small value.

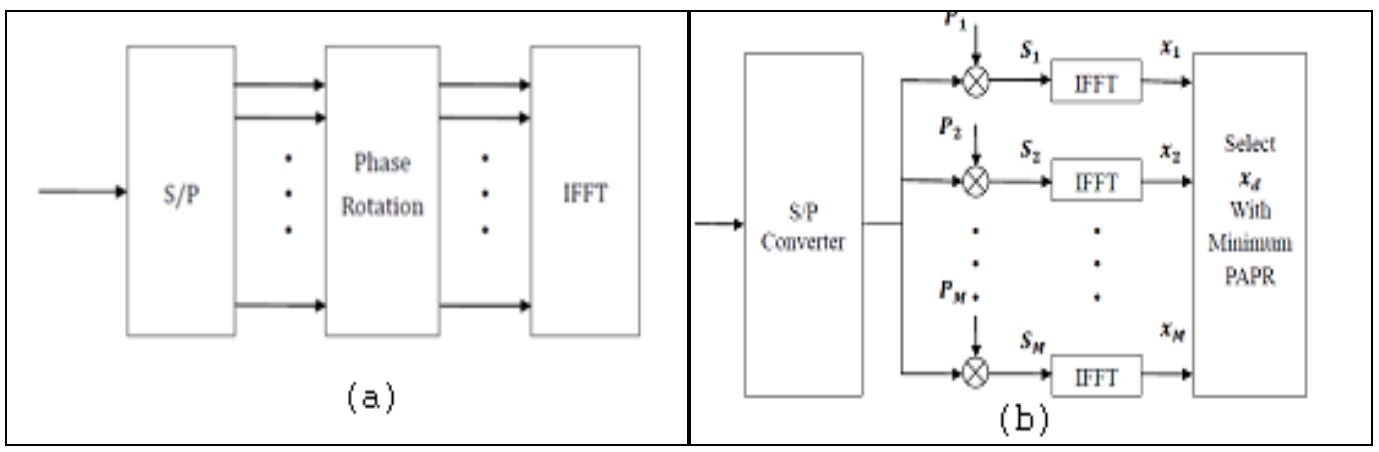

Figure 4 (a) Block diagram of phase rotation (b) principle of selected mapping.

This method can significantly improve the PAPR performance of OFDM system. Data blocks $\boldsymbol{S}_{\mathrm{m}}=\left[S_{m, 0}, S_{m, 1}, \ldots, S_{m, N-1}\right]^{T}, m=1,2, \ldots, M$ are statistically independent, assuming a single OFDM symbol, the CCDF probability of PAPR larger than a threshold is equals to $p$. The general probability of PAPR larger than a threshold for $k$ OFDM symbols can be expressed as $p^{\mathrm{K}}$. It can be verified that the new probability obtained by SLM algorithm is much smaller compared to the former. Data blocks $\boldsymbol{S}_{\mathrm{m}}$ are obtained by multiplying the original sequence with $M$ uncorrelated sequence $\boldsymbol{P}_{\mathrm{m}}$.

\subsection{Partial Transmit Sequence}

\subsubsection{Principle}

Partial Transmit Sequence (PTS) algorithm was first proposed by Muller S H and Huber J B [8] and [9], which is a technique for improving the statistics of a multi-carrier signal. The basic idea of partial transmit sequences algorithm is to divide the original OFDM sequence into several sub-sequences, and for each sub-sequence, multiplied by different weights until an optimum value is chosen. Fig. 5. is the block diagram of PTS algorithm. 


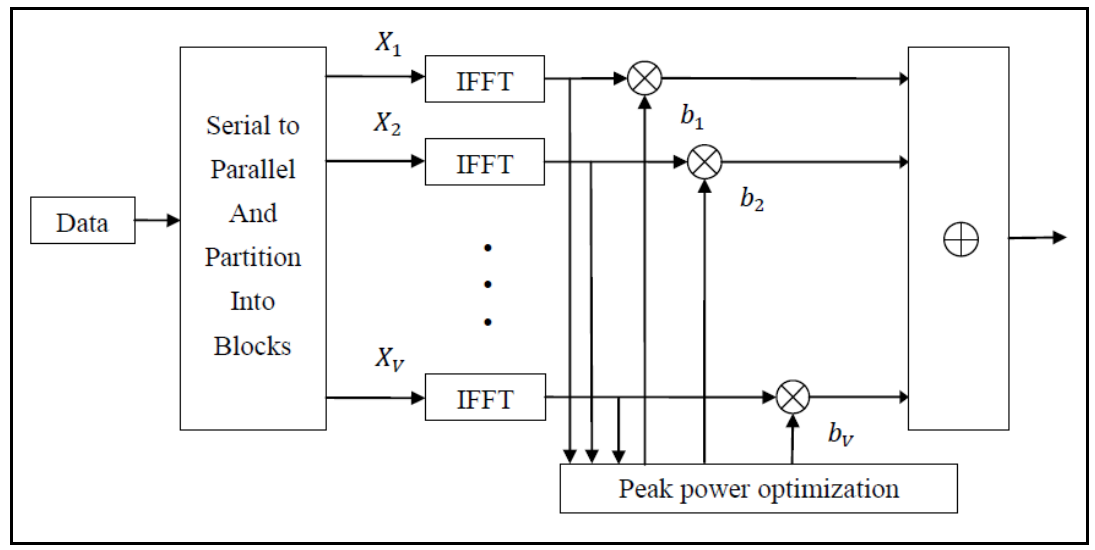

Figure 5 Block diagram of PTS algorithm

From the left side of diagram, we see that the data information in frequency domain $\mathbf{X}$ is separated into $\mathrm{V}$ non-overlapping sub-blocks and each sub-block vectors has the same size $N$. Hence, we know that for every sub-block, it contains $N / V$ nonzero elements and set the rest part to zero. Assume that these sub-blocks have the same size and no gap between each other, the sub-block vector is given by

$$
\widehat{\mathbf{X}}=\sum_{v=1}^{V} b_{v} \mathbf{X}_{v}
$$

where $b_{v}=e^{j \varphi v}\left(\varphi_{v} \in[0,2 \pi]\right), v=1,2, \ldots, V$ is a weighting factor used for phase rotation .The signal in time domain is obtained by applying IFFT operation on $X_{v}$, that is

$$
\hat{\mathbf{x}}=\operatorname{IFFT}(\widehat{\mathbf{X}})=\sum_{v=1}^{V} b_{v} \operatorname{IFFT}\left(\mathbf{X}_{v}\right)=\sum_{v=1}^{V} b_{v} \cdot \mathbf{x}_{v}
$$

Select one suitable factor combination $\mathbf{b}=\left[b_{1}, b_{2}, \ldots, b_{v}\right]$ which gives optimum result. The combination can be given by

$$
\mathbf{b}=\left[b_{1}, b_{2}, \ldots, b_{v}\right]=\arg \min _{\left(b_{1}, b_{2}, \ldots, b_{v}\right)}\left(\max _{1 \leq n \leq N}\left|\sum_{v=1}^{V} b_{v} x_{v}\right|^{2}\right)
$$

where $\arg \min (\cdot)$ is the judgment condition that output the minimum value of function. In this way we can find the best $\mathbf{b}$ so as to optimize the PAPR performance.

\subsection{Modified PTS Scheme}

Based on the discussion above, we had realized that the PAPR reduction performance and computational complexity of PTS algorithm is closely related to the sub-block partition and the value range of weighting factor.

\subsubsection{Sub-block partition scheme}

In PTS-OFDM system, there exist three seed sub-block partition schemes: adjacent partition, pseudo-random partition and interleaved partition. In Fig. 6. Graphs are shown for the illustration of the three partition schemes. From the figure we can see that adjacent partition is divide sequence into $V$ sub-blocks, for each one, it contains $N / V$ consecutive sub-carriers; In pseudo-random partition, each sub-carrier can be randomly assigned to any position of subblock with the length $V$; Interleaved partition also segments the sequence into $V$ sub-blocks but within each of them, sub-carriers are allocated in a space of $V$. The common point of these three different partition schemes is that each sub-carrier is only been assigned once, and the length of each sub-sequence is same. 
International Journal of Distributed and Parallel Systems (IJDPS) Vol.3, No.3, May 2012

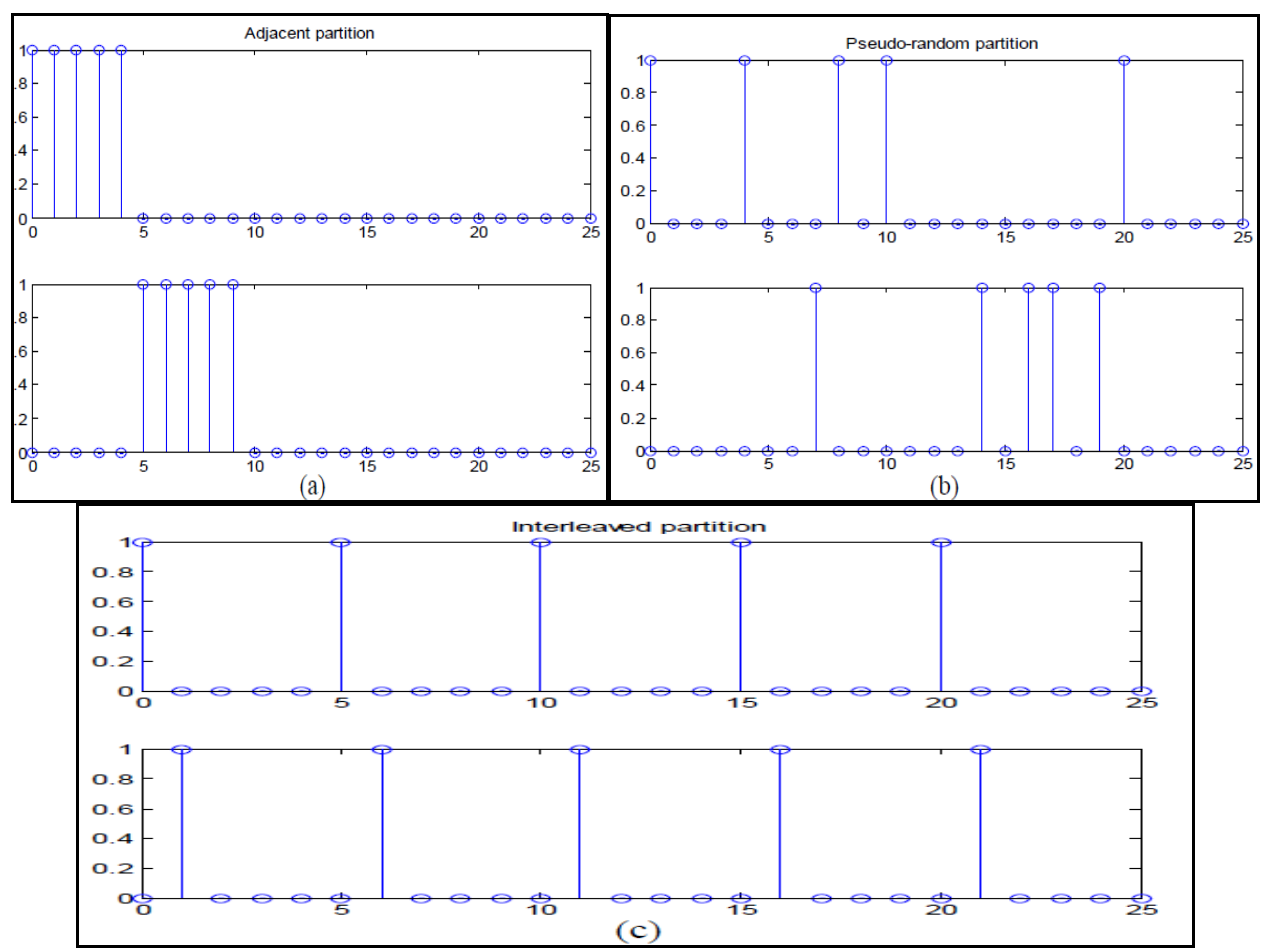

Figure 6 Illustration of different sub-block partition schemes: (a) adjacent partition, (b) pseudorandom partition, (c) interleaved partition.

\subsubsection{Effects on PAPR reduction in different sub-block partition schemes}

Fig.7. displays the PAPR reduction using PTS proposal with different sub-block partition schemes, in which QPSK is applied, $N=256$ and $V=1,2,3,4$, respectively. As shown in Fig. 7 the CCDF curve which is represented by dotted line using adjacent partition scheme, and solid line is plotted based on the pseudo-random partition scheme. The CCDF curves from left to right correspond to the case with sub-block number $V=4, V=3, V=2$, and $V=1$ respectively. As we can see from the graph, for each $V$ system performance of pseudo-random partition is superior by $0.5 \mathrm{~dB}$ (at minimum) to the one based on adjacent partition.

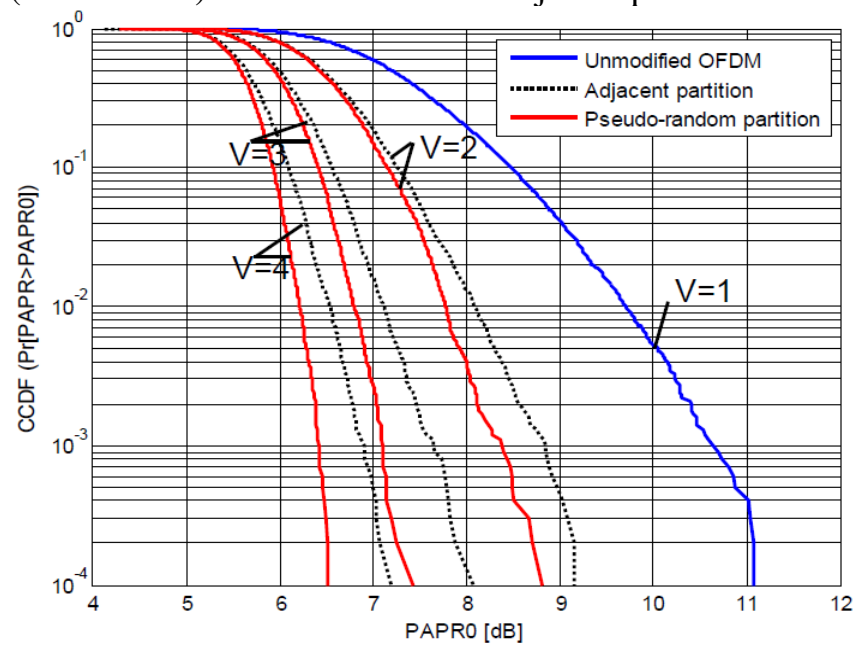

Figure 7 Comparison of PAPR reduction performances with difference sub-block partition schemes. 


\subsection{Comparison of SLM and PTS Algorithm}

SLM and PTS algorithms are two typical non-distortion techniques for reducing PAPR in OFDM system. In order to have error-free demodulation in the receiving end, side information must also be sent to the receiver. Hence, practical applications often require the use of some coding measures to protect information from being disturbed. Fig. 8. shows the simulation result of using SLM and PTS method to an OFDM system, separately. In PTS method, we set the number of sub-carriers $N=128$ and apply pseudo-random partition scheme for each carrier, adopting QPSK constellation mapping and weighting factor $b v \in \pm 1, \pm \mathrm{j}$; In SLM method, rotation factor $P m, n \in \pm 1, \pm j$. Based on the theory, we know that the IFFT calculation of these two methods is same when $V=M$, but for PTS method, it can provide more signal manifestations, thus, PTS method should provide a superior performance on PAPR reduction. In fact, this deduction is confirmed by simulation result. From Fig. 8. with the same CCDF probability $1 \%$ the PAPR value equals to $7 \mathrm{~dB}$ for PTS is $8.2 \mathrm{~dB}$ for SLM.

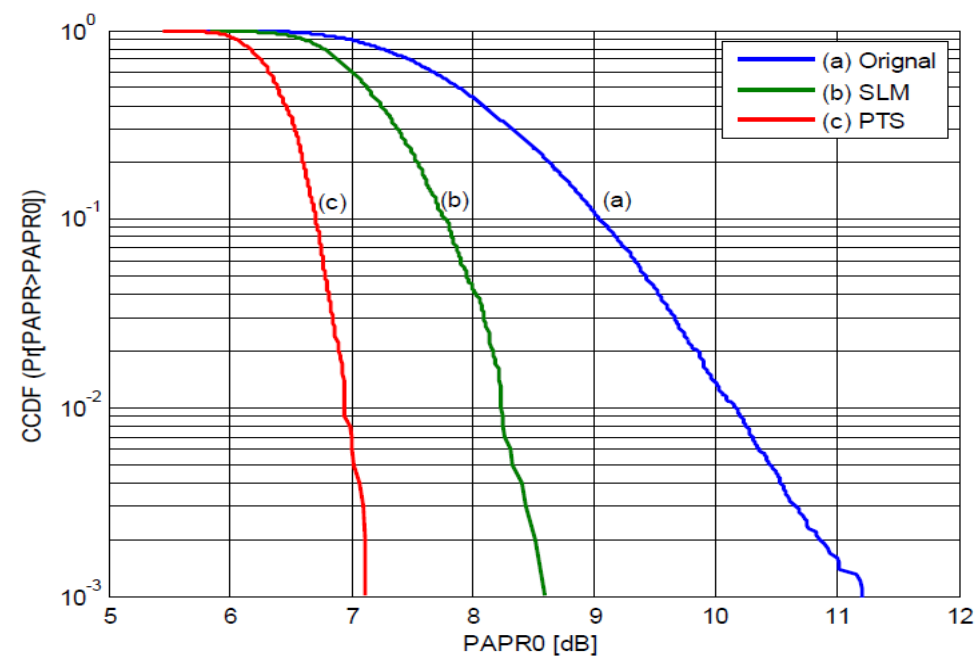

Figure 8 Comparison of PAPR reduction performances between PTS algorithm and SLM algorithm for PTS-OFDM system.

It shows clearly that PTS method provides a better PAPR reduction performance compared to SLM method. Thus, in practical applications, a tradeoff should be made between good performance and side information. From the discussion above, we can say that SLM algorithm is more suitable if system can tolerate more redundant information, otherwise, PTS algorithm is more acceptable when one requires less complexity.

\subsection{Suboptimal iterative algorithm}

The traditional PTS method is a traversal algorithm, which requires all possible phase values to be evaluated, that actually constrain the real application. For this reason, a suboptimal algorithm is introduced to reduce the number of phase patterns evaluation. It reduces the complexity and combines the advantage of PTS scheme on PAPR reduction performance. A small degree of degradation is observed compared to the optimal solution [9]. The following steps describe the algorithm:

1) Divide $N$ sub-carriers into $V$ non-overlapping sub-blocks.

2) Assume that $\mathbf{b}=b_{1}, b_{2}, \ldots, b_{v}=[1,1, \ldots, 1]$, and then calculate the PAPR value, $P A R x^{\prime}$, of an intermediate sequence $x^{\prime}$ in time domain. Initialize index $=1$, which represents the subscript of $b_{\text {index }}$. 
International Journal of Distributed and Parallel Systems (IJDPS) Vol.3, No.3, May 2012

3) Assume that $b_{\text {index }}=-1$, and calculate the PAPR, PARx, of this new sequence $x$.

4) If $P A R x>P A R x^{\prime}$, let $b_{\text {index }}=-1$, otherwise, $P x^{\prime}=P x$, and make index $=$ index +1 .

5) If index $<V+1$, go to step 3 , otherwise jump to step 6 . The iteration continues until index $=$

$V$.

6) Obtain sub-optimal weighting factor $\mathbf{b}$ and the corresponding PAPR is $\min \left(P x, P x^{\prime}\right)$.

Adopting iterative algorithm for searching suboptimal weighting factor $\mathbf{b}$, only $V$-steps calculation are needed. In each step, the IFFT calculation only be performed for corresponding one sub-block rather than calculate the all $V$ sub-blocks, by this means, it can reduce the complexity of the calculation significantly. The flow chart of suboptimal algorithm is shown in Fig. 9.

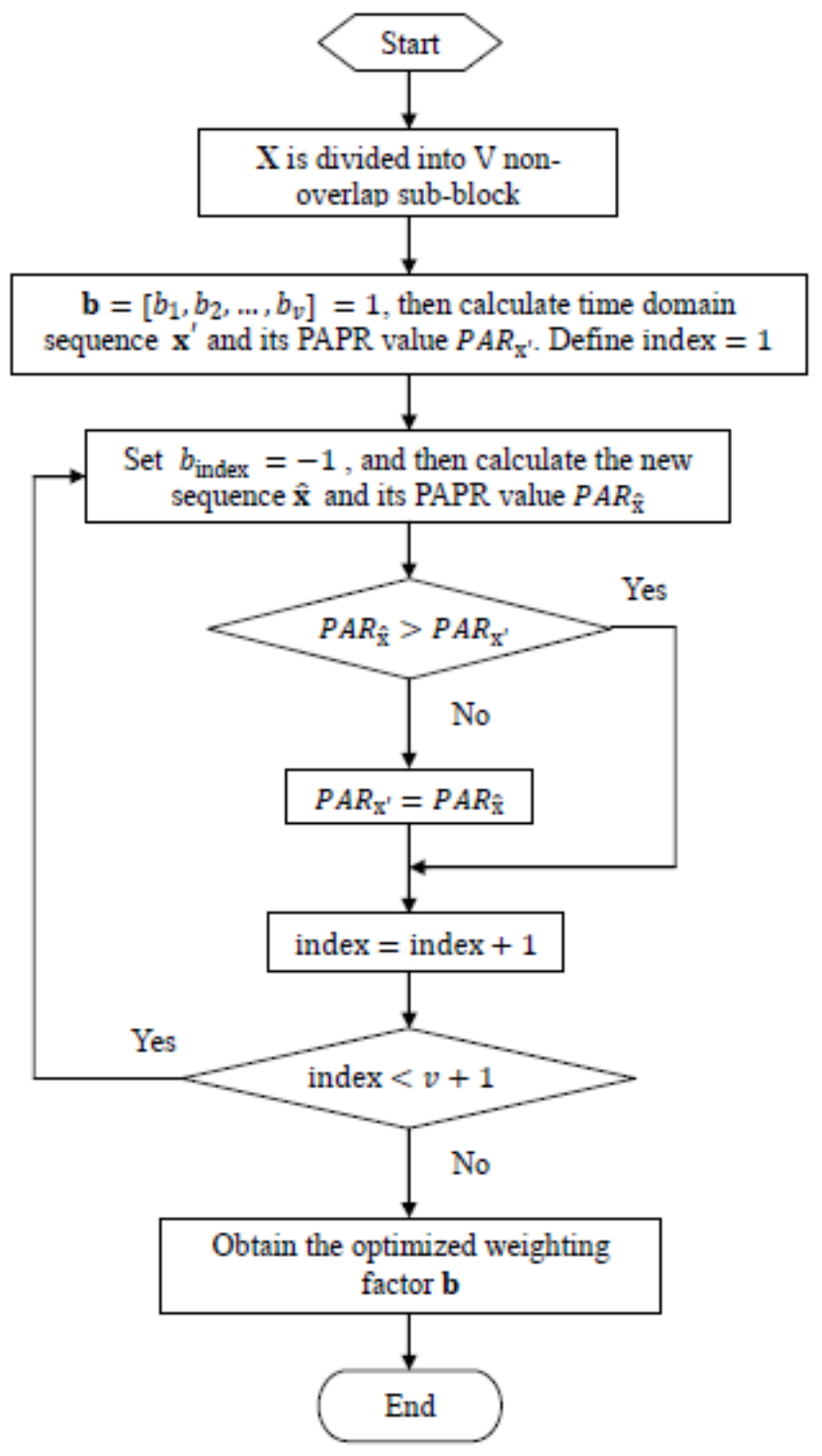

Figure 9 Flowchart of suboptimal iterative algorithm. 


\subsection{Comparison of iterative sub-optimization and optimization algorithms}

For PTS-OFDM system, simulation is carried out with the number of sub-carriers $\mathrm{N}=128$, and applying QPSK modulation, pseudo-random partition scheme is adopted for these two algorithms, and sub-block number takes $V=4$. For the convenient of comparison, the range of weighting factor is set as two for both cases, which is $b_{v}=[ \pm 1]$. Simulation results are shown in Fig. 10. and analyzed on the basis of observation. The CCDF curve of sub-optimization PTS algorithm lies in the middle of unmodified system's CCDF curve and CCDF curve processed by conventional PTS method and sub-optimization algorithm does not provide the best performance due to its inherent disadvantages. However, in real application, since sub-optimal algorithm can reduce system complexity greatly, we prefer to choose it instead of the conventional optimum algorithm.

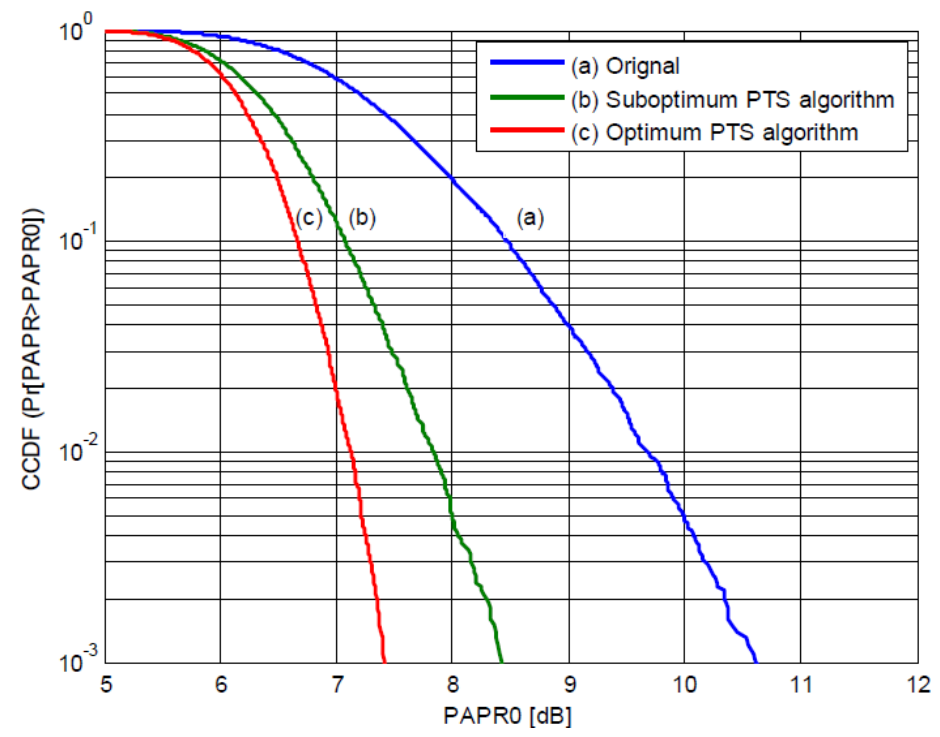

Figure 10 Comparison of PAPR reduction performances between iterative (sub-optimization) algorithm and optimization algorithm for PTS-OFDM system.

\section{CONCLUSIONS}

This paper provides an overview of Multiple-Input-Multiple-Output (MIMO) technology and Orthogonal-Frequency-Division-Multiplexing (OFDM). The focus of paper is that we investigate one of the bottleneck problems that exist in OFDM wireless communication system - high peak-average power ratio (PAPR) of OFDM signal, and discuss how to reduce it by different effective algorithms. The main contributions in this paper are listed below:

1. The comprehensive research and comparison are put forward for a variety of currently promising PAPR reduction methods quoted in the literature in this research area. Among these different proposals, we are mainly focusing on the signal scrambling technology, and verify the theoretical analysis by observing the MATLAB simulation results. At the same time, some meaningful guidance and conclusions are obtained through the comparative analysis of these simulation results as well.

2. In the signal scrambling technology, we study the method of selected mapping and partial transmit sequence. A series of detailed simulations were conducted for comparison and results were obtained of the two schemes for PAPR reduction in a complex system. This method has lots of merits, such as high coding rate and low redundancy, although it only optimizes the statistical characteristics of PAPR in OFDM system. To sum up the advantages and disadvantages of two algorithms it is pointed out that the trade offs for their respective 
International Journal of Distributed and Parallel Systems (IJDPS) Vol.3, No.3, May 2012

adaptation. For the inherent defect of traditional PTS algorithm, complex computing, a very effective iterative method is introduced to determine sub-optimal weighting factor for each subblock instead of conducting an ergodic searching so as to reduce the calculation complexity significantly. This sub-optimal algorithm gives a better approach to the real conditions in engineering practice by providing a compromise between the PAPR reduction performance and computational complexity.

\section{ACKNOWLEDGEMENTS}

I sincerely thank Prof.G.T Rao, E.C.E Department, G.V.P College of Engineering (Autonomous) for his immense support and valuable suggestions.

\section{REFERENCES}

[1] Young Kyun Kim, Ramjee Prasad, "What is 4G," in $4 G$ Roadmap and Emerging Communication Technologies, Artech House, 2006, pp. 12-13.

[2] Jaylath, A.D.S, Tellainbura, C, "Side Information in PAR Reduced PTS-OFDM Signals," Proceedings 14th IEEE Conference on Personal, Indoor and Mobile Radio Communications, Sept. 2003, vol. 1, PP. 226-230.

[3] Oh-Ju Kwon and Yeong-Ho Ha, "Multi-carrier PAP reduction method using sub-optimal PTS with threshold," IEEE Transactions on Broadcasting, June. 2003, vol. 49, no. 2, PP. 232-53 236

[4] Mohinder Jankiraman, "Peak to average power ratio," in Space-time codes and MIMO systems, Artech House, 2004, pp. 201.

[5] Christopher Gerald Santos, "Peak-to-Average power ratio reduction solutions for OFDM," Philippine Journal of ICT and Microelectronics, vol.1, no. 2, July 2002.

[6] Jones, A.E, Wilkinson, T.A, Barton, S.K, "Block coding scheme for reduction of peak to mean envelope power ratio of multicarrier transmission scheme," IEEE Electronic Letters, vol. 30, no. 25, Dec 1994, pp. 2098-2099.

[7] Davis, J.A, Jedwab, J, "Peak-to-Mean power control and error correction for OFDM transmission using Golay sequences and Reed-Muller codes," IEEE Electronic Letters, vol. 33, no. 4, Feb 1997, pp. 267-268.

[8] S. H. Muller, J. B. Huber, "OFDM with reduced peak-to-average power ratio by optimum combination of partial transmit sequence," IEEE Electronic Letters, Vol. 33, No. 5, Feb 1997, pp. 368-369.

[8] S. H. Muller, J. B. Huber, "A novel peak power reduction scheme for OFDM," The 8th IEEE International Symposium on Personal, Indoor and Mobile Radio Communications, Feb 1997, pp. 1090-1094.

[9] Leonard J. Cimini, Jr., Nelson R. Sollenberger, "Peak-to-Average power ratio reduction of an OFDM signal using partial transmit sequences," IEEE Electronic Letters, vol. 4, no. 3, Mar 2000, pp. 88-86.

[10] A.Ghassemi, T.A.., Gulliver, "A Low-Complexity PTS Based Radix FFT Method for PAPR Reduction in OFDM Systems," IEEE Trans. Signal Processing, vol.56, no.3, pp. 1161-1 166, March 2008.

[11] Tao Jiang and Yiyan Wu, "An Overview: Peak-to-Average Power Ratio Reduction Techniques for OFDM Signals", IEEE Transactions on Broadcasting, Vol. 54, No. 2, June 2008, pp. 257-267.

[12] Zhefeng Li and Xiang-Gen Xia, "PAPR Reduction for Space- Time-Frequency Coded MIMO-OFDM Systems using Chu Sequences", IEEE Transactions on Wireless Communications, Vol.7, No. 4, April 2008, pp. 1195-1202.

[13] Yung-Lyul Lee, Young-Hwan You, Won-Gi Jong-Ho Paik and Hyoung-Kyu Song "Peak-to-Average Power Ratio Reduction in MIMO OFDM System using Selective mapping", IEEE Communications Letters, Vol.7, No.12, December 2003, pp.575-577. 
[14] Jun Hou, Jianhua Ge and Jing Li, "Peak-to-Average Power Ratio Reduction of OFDM Signals using PTS Scheme with Low Computational Complexity", IEEE Transactions on Broadcasting, Vol.57, No.1, September 2010, pp. 143-148.

[15] Mohinder Jankiraman, "Multicarrier techniques," in Space-time codes and MIMO systems, Artech House, 2004, pp.201.

[16] Ben Lu and Xiaodong Wang, "Space-Time Code design in OFDM Systems", Proceedings of IEEE Global Telecommunications Conference, San Francisco, USA, December 2000, Vol. 2, pp.1000-1004.

[17] Mohinder Jankiraman, "Peak to average power ratio," in Space-time codes and MIMO systems, Artech House, 2004, pp. 201.

\section{Authors}

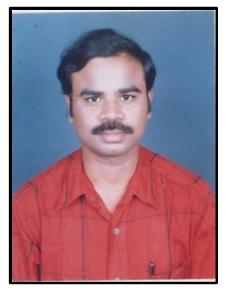

K.Srinivasa Rao received the B.Tech degree in Electronics and Communication Engineering from Nagarjuna University, and M.Tech degree in Instrumentation and Control Systems from J.N.T University College of Engineering. Kakinada, he has 9 years of teaching experience and is Sr.Assistant Professor of Electronics and Communication Engineering, G.V.P.College of Engineering for Women, Visakhapatnam. His research interests include Wireless Communications, Signal processing. He pursuing for Ph.D. from J.N.T.U. Kakinada.

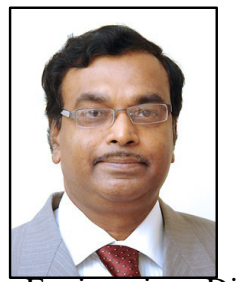

Dr.B.PrabhakaraRao received his B.Tech degree in Electronics and Communications Engineering. M.Tech degree in Electronics and Communication Systems from SVUniversity, Tirupati in 1979, 1981 respectively and received the Ph.D degree from IISC, Bangalore in 1995. Dr.B.Prabhakara Rao has more than 28 years of experience in teaching and 20 years of $\mathrm{R} \& \mathrm{D}$. He is an expert in Signal Processing \& Communications. He produced $7 \mathrm{PhD}$ 's and guiding $25 \mathrm{PhD}$ scholars. He held different positions in his career like Head of the Department, Vice Principal, in JNTU College of Engineering, Director (Institute of Science \& Technology) and Director of Evaluation; currently he is Director, Foreign University \& Alumni Relations in the Newly Established JNT University. He published more than 95 technical papers in National and International journals and conferences .Interests are in coding theory, information theory and signal processing with applications to wireless communications.

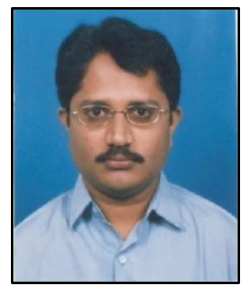

Dr.M.V.S.Sairam received the B.Tech degree in E.C.E from SRKR Engineering College in 1996, and M.Tech from Andhra University in 2002. He obtained his Ph.D degree from JNT University, kakinada in 2011. Now he is currently working Professor in GVP College of Engineering (Autonomous), Visakhapatnam, A.P., India. He is the author of text book "Principles of Communications". He is a member of ISTE and The Institution of Engineers. His research interests include Wireless Communications, Signal processing and coding theory. 